[0212-7199(2001) 18: 10; pp 517-520] ANALES DE MEDICINA INTERN Copyright $\odot 2001$ ARAN EDICIONES, S.A.

AN. MED. INTERNA (Madrid) Vol. 18, N. ${ }^{\circ} 10$, pp. 517-520, 2001

\section{Supervivencia y progresión de la enfermedad en 251 pacientes con infección por el VIH-1. Estudio del antígeno p24 y de la carga viral como marcadores pronóstico. Su valor a los 4 años de seguimiento}

\author{
M. RUBIO CABALLERO, C. RUBIO RIVAS, A. NOGUÉS BIAU, M. FALGUERA \\ SACREST, A. MANONELLES FERNÁNDEZ
}

Servicios de Medicina Interna y Análisis Clínicos (Sección de Microbiología).

Hospital Universitario Arnau de Vilanova. Lleida SURVIVAL AND PROGRESSION IN 251 PATIENTS WITH HIV-1
DISEASE. STUDY OF P24 ANTIGEN AND VIRAL LOAD AS SURRO -
GATE MARKERS. THEIR UTILITY IN 4 YEARS OF FOLLOW-UP

\section{RESUMEN}

Fundamento: Analizar el valor de los niveles plasmáticos de antígeno p24 y la carga viral (RNA, PCR), como marcadores pronóstico en una cohorte de pacientes infectados por el VIH-1, cuyo tiempo de seroconversión es desconocido.

Pacientes: Se incluyeron 251pacientes, la mayoría con terapia antirretroviral, que fueron asistidos de forma consecutiva en la Unidad VIH/SIDA del Servicio de Medicina Interna del Hospital Universitario Arnau de Vilanova de Lleida.

Métodos: Se hicieron estudios clínico-analíticos en el momento de inclusión (basal) y luego, cada 3 meses.

En relación al antígeno p24, se establecieron 3 grupos: Grupo I, $<20$ $\mathrm{pg} / \mathrm{mL}$; grupo II, $20-39 \mathrm{pg} / \mathrm{mL}$; grupo III, 40 o más $\mathrm{pg} / \mathrm{mL}$.

Se estudió la supervivencia y la progresión en relación con las cifras basales a lo largo de los 4 años.

En cuanto a la determinación del RNA por PCR, sólo se comparó con el antígeno p24 en la última fase del estudio ( $3^{\circ}$ y $4^{\circ}$ años), por razones técnicas.

El análisis de supervivencia se hizo con el método de Kaplan-Meier. El riesgo relativo se calculó por el modelo de riesgo proporcional de Cox.

Resultados: Durante los 48 meses de seguimiento fallecieron 55 pacientes. Había una diferencia estadísticamente significativa $(\mathrm{p}<0,0001)$ en la supervivencia entre el grupo I (ag p24 $<20 \mathrm{pg} / \mathrm{mL}$ ) y los otros 2 grupos. En cambio, no la había (p<0,57) en tre el grupo II (Ag p24 = 20-39 $\mathrm{pg} / \mathrm{mL})$ y el grupo III $(\mathrm{Ag}$ p $24=>40 \mathrm{pg} / \mathrm{mL})$.

Respecto al estudio de progresión, 34 pacientes lo hicieron. Nuevamente apreciamos una diferencia estadísticamente significativa $(\mathrm{p}=0,0039)$ entre el grupo I y los grupos II y III, pero no $(\mathrm{p}=0,37)$ entre el grupo II y el III.

La comparación de los niveles plasmáticos de antígeno p24 con la carga viral por PCR pone de manifiesto una gran disparidad de resultados.

Conclusiones: El nivel plasmático del antígeno p24 es un buen marcador pronóstico de supervivencia y de progresión a SIDA o muerte en enfermos infectados por el VIH-1 y su validez se prolonga por lo menos 4 años. Una cifra aislada $<20 \mathrm{pg} / \mathrm{mL}$ es un signo de mejor pronóstico.

No parece existir paralelismo entre los valores plasmáticos de antígeno p24 y la carga viral.

PALABRAS CLAVE: SIDA. Pronóstico. Supervivencia. Marcadores pronóstico. Antígeno p24.

\section{ABSTRACT}

Background: Prospective study of survival and AIDS or death pro gression in a cohort of 251 HIV infected patients whose seroconversion time is unknown, with a main objetive: To analyse p 24 antigen plasmatic levels and viral load as surrogate markers.

Patients: 251 patients were included, most of them undergoing anti retroviral therapy, and were followed-up consecutively in the HIV/AIDS Unity of Internal Medicine Service of the Hospital Universitario Arnau de Vilanova in Lleida.

Methods: We made clinical and analytical baseline studies and every 3 months thereafter.

Related to p24 antigen 3 group were established: group $1,<20$ $\mathrm{pg} / \mathrm{mL}$, group 2, 20-39 pg/mL, group 3, 40 or more $\mathrm{pg} / \mathrm{mL}$.

We studied survival and progression according to baseline levels over 4 year period.

Regard to viral load, we just compared this with p24 antigen in the last phase of the study (third and fourth year) for technical reasons.

Survival analysis was made by Kaplan-Meier estimation. Relative risk was calculated by Cox's proportional hazards model.

Results: During the 48 months of follow-up 55 patients died. AIDS progression risk or death was 4.8 times higher for the p24 antigen > = $40 \mathrm{pg} / \mathrm{mL}$ group than for the $\mathrm{p} 24$ antigen $<20 \mathrm{pg} / \mathrm{mL}$ one; the relative risk of patients with p24 antigen between 20-39 pg/mL was 2.5 times hig her than those included in the group of p24 antigen $<20 \mathrm{pg} / \mathrm{mL}$.

Related to progression study, 34 patients progressed. AIDS progres sion risk or death for p24 antigen $>=40 \mathrm{pg} / \mathrm{mL}$ group was 7.69 times higher compared with group 2 (p24 antigen levels between 20-39 $\mathrm{pg} / \mathrm{mL}$ ).

The comparison with viral load by PCR determination shows contro versial results.

Conclusions: p24 antigen plasma level is a good survival and AIDS progress or death surrogate markers in HIV infected patients, and it is useful for 4 years or more.

An isolated value $<20 \mathrm{pg} / \mathrm{mL}$ is a sign of good prognosis.

Parallelism between p24 antigen plasmatic level and viral load has not been observed.

KEY WORDS: AIDS. Prognosis. Survival. Surrogate markers. p24 anti gen.

Rubio Caballero M, Rubio Rivas C, Nogués Biau A, Falguera Sacrest M, Manonelles Fernández A. Supervivencia y progresión de la enfermedad en 251 pacientes con infección por el VIH-1. Estudio del antígeno p24 y de la carga viral como marcadores pronóstico. Su valor a los 4 años de seguimiento. An Med Interna (Madrid) 2001; 18: 517-520.

Trabajo aceptado: 21 de Mayo de 2001 


\section{INTRODUCCIÓN}

En los 20 años transcurridos desde el inicio de la epidemia del SIDA, en el comienzo de los años 80 , se ha constatado la diferente velocidad de progresión en los distintos pacientes. El $5 \%$ aproximadamente de los pacientes desarrollan un SIDA en 3 años de la infección (1). En cambio, el 12\% se mantendrán libres de SIDA más de 20 años (2). Esta variabilidad crea incertidumbre y dudas a la hora de iniciar un tratamiento, por lo que se ha tratado de encontrar marcadores útiles que nos puedan avisar de una rápida evolución. Estos marcadores pronóstico han ido cambiando a lo largo de los años.

El más firmemente establecido es el recuento de linfocitos CD4+, que ha condicionado los tratamientos, así como la profilaxis de las infecciones oportunistas (3).

El nivel plasmático del antígeno p24 ha tenido una vida azarosa. En un principio se valoró exclusivamente su positividad, que, aunque útil, dejaba mucho que desear. Luego se puso en duda su sensibilidad, ya que su determinación puede verse dificultada en presencia de exceso de anticuerpos antip24, con los que formaría complejos inmunes. Cuando la técnica consiguió superar esta dificultad (por acidificación o por calentamiento), surgió como gran rival la determinación de RNA (o ADN), que parece haberle vencido, ya que su indetectabilidad, sobre todo a partir de 1996, se ha convertido en el primer objetivo a alcanzar en el tratamiento de la infección por el VIH.

Sin embargo, algunos de nosotros dudamos de que el entierro precoz de la determinación del nivel plasmático del antígeno p24 haya sido correcto. En el estudio que aquí presentamos nos proponemos valorar su utilidad como marcador pronóstico de supervivencia, comparándolo, además, con el recuento de linfocitos CD4+ y con la determinación del RNA viral por PCR. En publicaciones anteriores (4-6) hemos demostrado su utilidad a los 3 años. En la actual confirmamos dicha utilidad a los 4 años.

\section{MATERIAL Y MÉTODOS}

\section{PACIENTES}

Se recogieron muestras de sangre de 251 pacientes adultos, consecutivos y no seleccionados, infectados por el VIH-1, asistidos en la Unidad VIH/SIDA del Servicio de Medicina Interna del Hospital Universitario Arnau de Vilanova de Lleida entre abril-94 y enero-95. Los pacientes prestaron su consentimiento informado. Se planificaron controles clínico-analíticos cada 3 meses. De 7 pacientes solo se obtuvo la visita inicial y la determinación basal, por lo que han sido excluidos de los resultados.

Más de un control se obtuvo en 244 pacientes, aunque 47 de ellos fueron controlados de forma irregular. De acuerdo con los criterios de CDC 1993 (7), los pacientes estaban distribuidos de la siguiente forma: estadio A, 140 pacientes; estadio B, 53; estadio C, 51.

Ciento sesenta y nueve pacientes estaban tomando tratamiento antirretroviral, inicialmente con inhibidores de la transcriptasa inversa, bien como monoterapia o como asociación de dos fármacos; a lo largo de los 4 años de estudio, el tratamiento se adaptó a las normas generalmente aceptadas en esa época, tomando como referencia la cifra de linfocitos CD4 en los dos primeros años y el valor de la carga viral en los otros dos.
Según el valor inicial del antígeno p24, se establecieron de forma arbitraria 3 grupos: el grupo I, compuesto por 173 pacientes que tenían $<20 \mathrm{pg} / \mathrm{mL}$; el grupo II lo componían 27 pacientes con antígeno p24 entre 20 y $39 \mathrm{pg} / \mathrm{mL}$; el grupo III estaba formado por 51 pacientes con p24>40 pg/mL.

El estudio de progresión a SIDA o muerte sólo se ha hecho en los 151 pacientes que en el inicio no estaban en estadio $\mathrm{C} \mathrm{y}$ que, además, se les siguió un control regular.

\section{MÉTODO}

La determinación del antígeno p24 se realizó por técnica de microelisa (VIRONOSTIKA HIV-1 Antigen. Organon Teknika N.V. Tornhont. Bélgica), siguiendo las indicaciones del fabricante. La sensibilidad del método es de $5 \mathrm{pg} / \mathrm{mL}$.

La carga viral se determinó por técnica de PCR (Amplicor. Roche), siendo el límite de detección de 500 copias $/ \mathrm{mL}$.

\section{ANÁLISIS ESTADÍSTICO}

El estudio de los tiempos de supervivencia y de progresión y la construcción de las curvas correspondientes se realizó por el método de Kaplan-Meier. Los grupos se compararon mediante la prueba de Mantel-Haenszel o prueba de log-rank. El riesgo relativo (RR) y el intervalo de confianza (IC) del 95\% se calcularon por el método de regresión de riesgos proporcionales de Cox. El nivel de significación se ha establecido en $\mathrm{p}<0,05$.

\section{RESULTADOS}

Durante 48 meses se estudiaron 244 pacientes con infección por VIH-1. El recuento inicial de linfocitos CD4 fue de media 377,102 (rango 0,8-1350). El seguimiento de 54 pacientes se perdió a lo largo de los 48 meses. 55 pacientes fallecieron en algún momento del seguimiento.

De forma arbitraria se establecieron 3 grupos, según el valor inicial del antígeno p24. El grupo I con p24 $<20$ pg/mL constaba de 173 pacientes, de los que fallecieron 22; la media de supervivencia fue de 44 meses (IC 95\%, 42-46). En comparación con el grupo II se obtuvo un logrank de 5,23, estadísticamente significativo $(\mathrm{p}=0,02)$. Respecto al grupo III, el logrank fue de 33,87 ( $\mathrm{p}<0,0001)$.

El grupo II con p24 20-39 pg/mL tenía 27 pacientes, de los que fallecieron 11; la media de supervivencia fue de 36 meses (IC 95\%, 30-43). En comparación con el grupo III el logrank fue de 2,69, que, aunque muestra una mayor supervivencia, no es estadísticamente significativo $(\mathrm{p}=0,1)$.

El grupo III con p24 =>40 pg/mL estaba formado por 51 pacientes, de los que fallecieron 22 ; la media de supervivencia fue de 32 meses (IC 95\%, 26-37).

En la figura 1 se presenta la curva de supervivencia de Kaplan-Meier de los 3 grupos. Los pacientes con antígeno p24 mayor tienen un pronóstico significativamente peor que los pacientes con una cifra menor. El test logrank de los 3 grupos fue de 33,24, estadísticamente significativo $(\mathrm{p}<0,0001)$. Comparando los grupos 2 a 2, entre el grupo I y el II, el logrank fue de $5,22(\mathrm{p}=0,02)$; entre el grupo I y el III, el logrank fue de 33,87 ( $\mathrm{p}<0,01)$; entre el grupo II y el III, el logrank fue de $2,69(\mathrm{p}=0,1)$. 


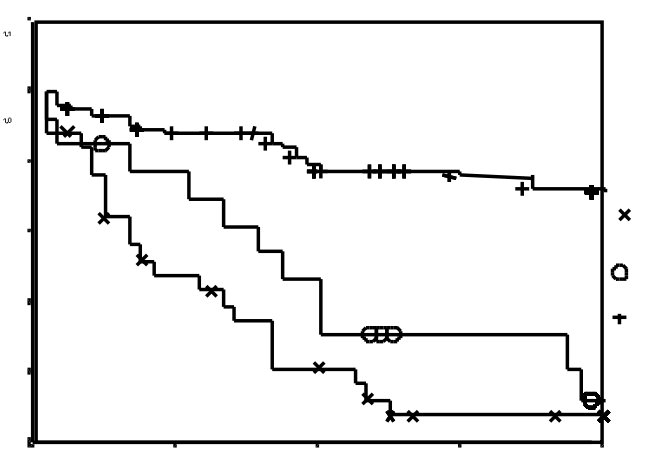

Fig. 1. Curvas de supervivencia de Kaplan-M eier según antígeno p24 basal.

El riesgo relativo de muerte fue de 2,5 veces superior en el grupo II respecto al grupo I y de 4,8 veces en el grupo III que en el I.

En relación con el cálculo de progresión, hemos suprimido 51 pacientes que tenían criterios de SIDA al inicio y 49 en los que no se pudo seguir un control regular, por lo que los cálculos se han hecho sobre los 151 restantes.

En la figura 2 se representa la curva de Kaplan-Meier de los 3 grupos, de acuerdo con el valor basal de p24. Es evidente la diferencia entre el grupo I y los otros 2, entre los cuales hay una gran coincidencia. En el grupo I hubo 108 pacientes, 18 de los cuales progresaron a SIDA o fallecieron en una media de 46 meses (IC 95\%, 44-47) En el grupo II se registraron 8 progresiones, entre 17 pacientes, con una media de 39 meses (IC 95\%, 31-46). En el grupo 3 había 26 pacientes, progresaron 8 y la media fue de 40 meses (IC 95\% 34-46). Comparándolos de 2 en 2, entre el grupo III y el I, el logrank fue de $7,69(\mathrm{p}=0,02)$; entre el grupo III y el II, el logrank fue de 6 $(p=0,01)$; entre el grupo II y el I, logrank $0,00(p=0,98)$.

En cuanto a la carga viral, se ha ido introduciendo en nuestra Unidad desde finales de 1996, por lo que solo disponemos de 125 pacientes en los que se han realizado 521 determinaciones simultáneas de ésta y de antígeno p24 en algún control del tercer y cuarto años de estudio.

En 219 casos $(42,03 \%)$, ambos parámetros fueron indetectables. En 83 (15,93\%), ambos estaban elevados. Sumando

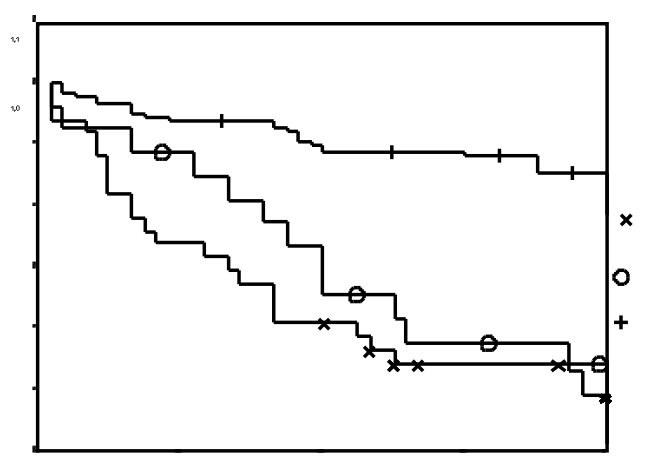

Fig. 2. Curvas de Kaplan-Meier. Progresión según antígeno p24 basal. ambos grupos, en 302 casos $(57,96 \%)$ se obtuvieron resultados más o menos coincidentes de los dos parámetros. En 219 casos $(42,03 \%)$ no hubo coincidencia en los resultados; en $160(30,71 \%)$, la CV fue positiva, a pesar de un antígeno p24 indetectable: en 59 (11,32\%), la CV fue indetectable, con un antígeno p24 alto. Estos resultados se expresan en la tabla I.

$$
\text { TABLA I }
$$

COM PARACIÓ N ENTRE CARGA VIRAL (CV) Y ANTÍGENO P24

\begin{tabular}{lcc}
\hline & CV indetectable & CV alta \\
\hline p24 indetectable & $219(42,03 \%)$ & $160(30,71 \%)$ \\
P24 ALTO & $59(11,32 \%)$ & $83(15,93 \%)$ \\
\hline
\end{tabular}

\section{DISCUSIÓN}

En este trabajo, presentamos nuestro estudio durante 4 años del valor del nivel plasmático del antígeno p24 como indicador del riesgo de muerte o de progresión a SIDA en pacientes infectados por el VIH-1. Este es un dato que manejamos en la práctica clínica y que puede ayudarnos en el momento de tomar la decisión de iniciar o modificar un tratamiento.

El presente estudio muestra que, a lo largo de 4 años, un nivel plasmático de antígeno p24 menor de 40 pg/ml se asocia con una mayor supervivencia y que ésta es aún mayor si los niveles plasmáticos están por debajo de 20 pg/ml. En cambio, en lo referente a la progresión a SIDA, las diferencias solo se establecen en la frontera de los $20 \mathrm{pg} / \mathrm{mL}$, siendo semejante para los situados entre 39 y $20 \mathrm{pg} / \mathrm{ml}$ que para los que muestran $40 \mathrm{pg} / \mathrm{mL}$ o más.

La antigenemia p24 ha sido usada desde hace tiempo para valorar la progresión de la infección por el VIH $(8,9)$. Sin embargo, la producción de complejos antígeno-anticuerpo hacían que su determinación no tuviera suficiente sensibilidad (10). Para superar el problema se ha recurrido a técnicas de disociación de complejos inmunes por acidificación, tratamiento con álkali o ebullición (11-14). La sensibilidad puede mejorarse por amplificación con ELISA (15).

En los últimos años se ha ido introduciendo la determinación de los niveles de RNA viral (carga viral, CV) en la práctica médica [16] y se han publicado estudios que demuestran una relación entre los niveles plasmáticos de RNA viral y la progresión a SIDA o la supervivencia (17-21). En uno de estos artículos reseñados (19) se insiste en subrayar que la antigenemia p24 no siempre se correlaciona apropiadamente con la progresión de la enfermedad. No obstante, tampoco la determinación de la carga viral se ha librado de la crítica (22).

Por otra parte, el ya célebre artículo de JW Mellors y cols. (23) concluye que el pronóstico de los pacientes VIH+ se define con más seguridad combinando las medidas de RNA-VIH1 en plasma con la de linfocitos CD4+.

En el mismo sentido que Mellors, nosotros también encontramos ventajas en la combinación de la determinación de antígeno p24 y de linfocitos CD4+.

Recientemente se ha publicado un estudio comparativo, en donde se demuestra que la sensibilidad del test para antígeno p24 por ELISA es semejante a la detección de RNA VIH-1 por PCR (24). Los mismos autores, en un artículo posterior (25), demuestran la utilidad de la antigenemia para monitori- 
zar la terapia antirretroviral y la similitud de sus curvas con las de RNA. Los cambios en la concentración plasmática del antígeno p24 también han servido para evaluar la eficacia de un tratamiento (25). Nosotros también hemos publicado anteriormente nuestros resultados respecto al pronóstico de supervivencia (4) y de progresión (5) en 2 y 3 años (6).

Respecto a la comparación de antígeno p24 y CV, nuestros resultados difieren de los de Schüpbach y cols., aunque puede haber existido una selección no intencionada por nuestra parte. Comparando los valores individuales apreciamos que, si con frecuencia coinciden niveles indetectables de ambos, no es excepcional encontrar discrepancias. En un reciente trabajo presentado en la VII Conferencia Europea sobre aspectos clínicos y tratamiento de la infección por VIH (26) se señala una posible correlación entre carga viral y antigenemia p24; nosotros no encontramos dicha correlación, aunque tampoco podemos señalar la superioridad de un marcador pronóstico sobre el otro.

En el presente tabajo, hemos querido demostrar que, a lo largo de 4 años, la determinación de antígeno p24 es útil también para predecir el riesgo de progresión y de muerte, considerando, bien un valor aislado o la sucesión de controles periódicos, en una cohorte de pacientes no seleccionados, con tiempo de seroconversión desconocido y con distintos tratamientos antirretrovirales. Además, hemos evidenciado que es un marcador pronóstico independiente de los linfocitos CD4+. La adopción del punto de corte de los 3 grupos en 20 y 40 pg/ $\mathrm{mL}$ ha sido arbitraria. La población estudiada no es muy numerosa y el tiempo de seguimiento es reducido (48 meses); sin embargo, consideramos que ambos son suficientes para dar valor a nuestra tesis.

\section{Bibliografía}

1. Phair J, Jacobson L, Detels R, Rinaldo C, Saah A, Schrager L, et al. Acquired immune deficiency syndrome ocurring within 5 years of infection with human immunodeficiency virus type-1: the Multicenter AIDS Cohort Study. J Acquir immune Defic Syndr 1992; 5: 490-6.

2. Sheppard HW, Lang W, Ascher MS, Vittinghoff E, Winkelstein W. The characterization of non-progressors: long-term HIV-1 infection with stable CD4+ T-cell levels. AIDS 1993; 7: 1159-66.

3. Grupo de Estudio de Sida (GESIDA). Recomendaciones sobre tratamiento antirretroviral. Med Clin (Barc) 1998; 110: 109-16.

4. Rubio Caballero M, Nogués Biau A, Falguera Sacrest M, Puig Ganau T, Lorente L. Valor de la cuantificación plasmática del antígeno p24 como marcador pronóstico de supervivencia en una cohorte de 251 pacientes VIH+. An Med Interna (Madrid) 1998; 15: 647-9.

5. Rubio Caballero M, Nogués Biau A, Falguera Sacrest M, Puig Ganau T. Marcadores pronóstico de progresión en la infección por VIH. Estudio del antígeno p24 en una cohorte de 251 pacientes. An Med Interna (Madrid) 1999; 16: 447-50.

6. Rubio Caballero M, Nogués Biau A, Falguera Sacrest M, Puig Ganau T, Lecha Cano N, Rubio Rivas C. Marcadores pronóstico de supervivencia y de progresión en la infección por el VIH-1. Estudio de linfocitos CD4+, antígeno p24 y carga viral durante 3 años en una cohorte de 251 pacientes. An Med Interna (Madrid) 2000; 17: 533-7.

7. Centers for Disease Control and Prevention: 1993 Revised classification system for HIV infection and expanded surveillance case definition for AIDS among adolescent and adults. MMWR 1992, 41: 1-19.

8. Allain JP, Laurian Y, Senn D. Serological markers in early stages of human immunodeficiency virus infection in haemophiliacs. Lancet 1986; ii: 1233-6.

9. Lange JMA, Paul DA, Huisman HG, de Wolf F, Van der Berg H,Coutinho RA, et al. Persistent HIV antigaenemia and decline of HIV core antibodies associated with transition to AIDS. Br Med J 1986; 293: 1459-62.

10. Lange JMA, Paul DA, de Wolf F, Coutinho RA, Goudsmit J. Viral gene expression, antibody production and immune complex formation in human immunodeficiency virus infection. AIDS 1987; 1: 15-20.

11. Kageyama S, Yamada O, Mohammad SS, Hama S, Hattori N, Asanaka $\mathrm{M}$, et al. An improved method for the detection of HIV antigen in the blood of carriers. J Virol Methods 1988; 22: 125-31.

12. Hyman J, Lockwood D, Holody T, Youngbar P. Base dissociation assay (BDA), a simplified method for immune-complex dissociation and detection of HIV p24. IX International Conference on AIDS / IV STD World Congress. Berlin, June 1993 (abstract PO-A31-0757).

13. Denis R, Henrard JF, Phillips LR, Muenz WA, Blattner DW, Eyster ME, et al. Natural History of HIV-1 Cell-Free Viremia. JAMA, 1995, 274: 554-558.

14. Schüpbach J, Böni J. Quantitative and sensitive detection of immunecomplexed and free HIV antigen after boiling of serum. J Virol Methods 1993; 43: 247-56.

15. Schüpbach J, Tomasik Z, Böni J, Kind C, and the Swiss Neonatal HIV
Study Group. Sensitive, reliable and cost-efficient diagnosis of pediatric HIV-1 infection by antigen p24 testing of plasma after heat-mediated immune-complex dissociation. FEMS Symposium on Recent Advances in the Diagnosis of Viral Diseases. Istanbul, July 1995 (abstract OP 11).

16. Mulder J, McKinney N, Christopherson C, Sninsky J, Greenfield L, Kwok S. Rapid and simple PCR assay for quantitation of human immunodeficiency virus type 1 RNA plasma: application to acute retroviral infection. J Clin Microbiol 1994; 32: 292-303.

17. Mellors JW, Kingsley LA, Rinaldo CR, Todd JA, Hoo BS, Kokka RP, et al. Quantitation of HIV-1 RNA in plasma predicts outcome after seroconversion. Ann Intern Med 1995; 122: 573-9.

18. O'Brien WA, Hartigan PM, Martin D, Esinhart J, Hill A, Benoit S, et al. Changes in plasma HIV-1 RNA and CD4+ lymphocyte counts and the risk of progression to AIDS. N Engl J Med 1996; 334: 426-31.

19. Ruiz L, Romeu J, Clotet B, Balagué M, Cabrera C, Sirera G, et al. Quantitative HIV-1 RNA as a marker of clinical stability and survival in a cohort of 302 patients with a mean CD4 cell count of 300 x 106 / 1 . AIDS 1996; 10: F39-F44.

20. Romeu J, Balagué M, Ruiz L, Marfil S, Puig T, Arnó A, et al. Valor de la carga viral del VIH-1 y de los linfocitos CD4+ como determinantes de la progresión a sida y de la supervivencia. Med Clin (Barc) 1998; 110: 761-7.

21. Mellors JW, Rinaldo CR, Gupta P, White RM, Todd JA, Kingsley LA. Prognosis in HIV-1 infection predicted by the quantity of virus in plasma. Science 1996; 272: 1167-70.

22. Fessel WJ. Human immunodeficiency virus (HIV) RNA in plasma as the preferred target for therapy in patients with infection: a critique. CID 1997; 24: 116-22.

23. Mellors JW, Muñoz A, Giorgi JV, Margolick JB, Tassoni CJ, Gupta P, et al. Plasma Viral Load and CD4+ Lymphocytes as Prognostic Markers of HIV-1 Infection. Ann Intern Med 1997; 126: 946-54.

24. Jörg Schüpbach, Markus Flepp, Dora Pontelli, Zuzana Tomasik, Ruedi Lüthy, Jürg Böni. Heat-mediated immune complex dissociation and enzyme-linked immunosorbent assay signal amplification render p24 antigen detection in plasma as sensitive as HIV-1 RNA detection by polymerase chain reaction. AIDS 1996; 10: 1085-90.

25. Jürg Böni, Milos Opravil, Zuzana Tomasik, Madeleine Rothen, Leslie Bisset, Peter J Grob, et al. Simple monitoring of antiretroviral therapy with a signal-amplification-boosted HIV-1 p24 antigen assay with heatdenatured plasma. AIDS 1997; 11: F47-F52.

26. de Jong MD, Vella S, Carr A, Boucher CAB, Imrie A, French M, et al. High-Dose Nevirapine in Previously Untreated Human Immunodeficiency Virus Type1-Infected Persons Does Not Result in Sustained Supression of Viral Replication. J of Infec Dis 1997; 175: 966-70.

27. Sabin CA, Devereux H, Phillips AN, Lee CA, Loveday C. The relationship between p24 antigen, HIV RNA level and disease progression in haemophilic individuals infected with HIV. 7th European Conference on Clinical Aspects and Treatment of HIV-Infection.- Lisbon October 1999. 